\title{
Unique Extension of Peritonsillar Abscess to the Masticator Space Encountered in the Early Phase of Antiviral Therapy for Hepatitis C
}

Hiroshi Hidaka $^{1 *}$, Takahiro Suzuki ${ }^{1}$, Eiichi Ishida ${ }^{1}$, RisakoKakuta ${ }^{1,2}$ and Hisakazu Yano ${ }^{2}$

${ }^{1}$ Department of Otolaryngology-Head and Neck Surgery, Tohoku University, Japan

${ }^{2}$ Department of Infection Control and Laboratory Diagnostics, Tohoku University, Japan

\begin{abstract}
Background: To describe a unique case of peritonsillar abscess showing peculiar extension to the masticator space, encountered in the early phase of telaprevir therapy in combination with peginterferonalfa- $2 \mathrm{~b}$ and ribavirin for hepatitis $\mathrm{C}$ virus $(\mathrm{HCV})$ infection.

Methods: Review of clinical case records, including computed tomography and surgical approaches.

Results: A 64-year-old woman was taking medications for HCV, including telaprevir therapy in combination with peginterferonalfa- $2 \mathrm{~b}$ and ribavirin every one week. She was also receiving medical treatment for diabetes mellitus. Her symptoms started 3 days after the commencement of antiviral therapy. She underwent surgery for drainage of the abscess, including endoscopy-assisted intraoral drainage and quinsy tonsillectomy.

Conclusions: It is important to bear in mind that a patient suffering from multiple systemic diseases may display unique clinical presentations with head and neck infections. Endoscopy-assisted intraoral drainageof the lateral masticator space was less invasive than other approaches, obviating the need for identifying the facial nerve.
\end{abstract}

Keywords: Peritonsillar abscess; Masticator space; Deep neck infection; Antiviral therapy, Telaprevir; Peginterferon; Ribavirin; Drainage; Endoscopy; Quinsy tonsillectomy

\section{Introduction}

Deep neck infection is a serious disorder that often spreads to other organs and sometimes proves fatal. Although the pattern of spread varies among patients, a relatively constant trend in the distribution into spaces seems evident because of the relationship of the cervical fascia, which directs and limits the spread of these infections $[1,2]$. Peritonsillar abscess is the most common type of deep neck infection, and sometimes extends to the lower cervical space via the parapharyngeal space [1].On the other hand, the masticator space is the most prevalent site of spread from odontogenic infection [3-5]. We report a unique case of peritonsillar abscess that showed an unusual extension to the masticator space via the parotid space, encountered after telaprevir therapy for hepatitis $\mathrm{C}$ in combination with peginterferonalfa- $2 \mathrm{~b}$ and ribavirin. The patient's abscess was successfully drained bythe intraoral approach using an endoscope.

The predominant organisms of the peritonsillar abscess are Streptococcus species and anaerobes such as Fusobacterium or Prevotella species [1,6,7]. Specifically, the Streptococcus milleri group (SMG, also referred to as the "Streptococcus anginosus group") has been reported to be the most common isolates of the Streptococcus species [1,7]. In bacterial culture analysis, Streptococcus constellatus was isolated in the current case, which is a member of the SMG.

\section{Case report}

A 64-year-oldwoman presented to our department with a 5-day history of pharyngeal pain and left-sided facial swelling. The patient had been receiving anti-virus therapy for hepatitis $\mathrm{C}$, including telaprevir therapy $(2250 \mathrm{mg})$ in combination with peginterferonalfa- $2 \mathrm{~b}(100 \mu \mathrm{g})$, and ribavirin $(600 \mathrm{mg})$ every one week for 1 year. The present symptoms occurred just 3 days after the 11thround of combined antiviral therapy. In the early phase of initiation of the patient's combined telaprevir therapy, she had suffered from telaprevir-related dermatitis, and had been prescribed prednisolone $(20 \mathrm{mg} /$ day $)$. Furthermore, she had also been prescribed oral hypoglycemic agents (glimepiride, $4 \mathrm{mg} /$ day; sitagliptin phosphate hydrate, $50 \mathrm{mg} /$ day; buformine hydrochloride, $100 \mathrm{mg} /$ day) because of steroid-induced diabetes mellitus for 1 month.

On physical examination, mild trismus and swelling of the left peritonsillar tissues with bulging of the tonsillar pillar were observed in addition to the facial swelling. Flexible laryngo scopy revealed an erythematous oropharynx with extension to the larynx. The white blood cell (WBC) count was 3,300 cells $/ \mathrm{mm}^{3}$, red blood cell $(\mathrm{RBC})$ count was $302 \times 10^{4} / \mu \mathrm{l}$, hemoglobin was $9.6 \mathrm{~g} / \mathrm{dl}$, platelet count was 42,000/l, C-reactive protein level was $20 \mathrm{mg} / \mathrm{dl}$, amylase was $589 \mathrm{U} / \mathrm{l}$, fasting blood sugar (FBS) level was $357 \mathrm{mg} / \mathrm{dl}$, and hemoglobin Alc was $10.9 \%$. Contrast-enhanced computed tomography (CT) revealed an abscess in the left parapharyngeal space, which extended to the lateral masticator space via the parotid space (Figure 1).

According to the above-mentioned findings, the patient was diagnosed with left peritonsillar abscess complicated with a unique extension from the parapharyngeal space to the lateral masticator space. Surgical drainage was thus performed via an intraoral approach under general anesthesia followed by tracheotomy. First, a $3-\mathrm{cm}$ incision was made in the right gingival-buccal mucosa, followed by dissection along the ramus of the mandible, assisted by an endoscope (Figure 2 ). By incising the laterally retracted mandibular periosteum and cervical fascia, abscess drainage from the lateral masticator space was

*Corresponding author: Hiroshi Hidaka, Department of Otolaryngology-HNS Tohoku University Graduate School of Medicine, 1-1 Seiryomachi, Aoba-ku, Sendai 980-8575, Japan, Tel: +81-22-717-7304; Fax: +81-22-717-7307; E-mail: ZAY00015@nifty.com

Received April 17, 2013; Accepted June 01, 2013; Published June 05, 2013

Citation: Hidaka H, Suzuki T, Ishida E, RisakoKakuta, Yano H (2013) Unique Extension of Peritonsillar Abscess to the Masticator Space Encountered in the Early Phase of Antiviral Therapy for Hepatitis C. Clin Microbial 2: 117. doi:10.4172/23275073.1000117

Copyright: ( 2013 Hidaka H, et al. This is an open-access article distributed unde the terms of the Creative Commons Attribution License, which permits unrestricted use, distribution, and reproduction in any medium, provided the original author and source are credited. 

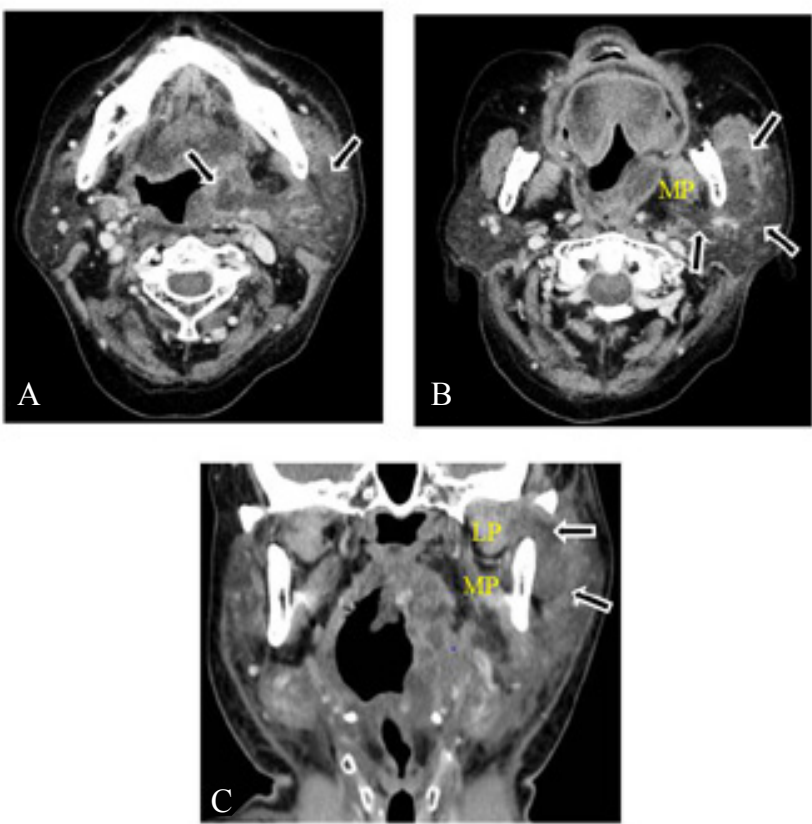

Figure 1: A)Pre-operative, axial enhanced computed tomography (CT) at the level of the mandible, demonstrating an abscess of the left parapharyngeal space extending into the parotid space (arrows). B) A more caudal CT at the level of the mandibular ramus, showing the abscess extending to the lateral masticator space (arrows). The abscess seems to extend along the parotid space and/or posterior oblique line of the mandibular ramus. Note that the abscess does not extend into the medial pterygoid muscle (MP). C)Coronal CT showing the abscess extending from the parapharyngeal space to the lateral masticator space (arrows). Note that the abscess does not extend into the medial (MP) or lateral pterygoid muscle (LP).

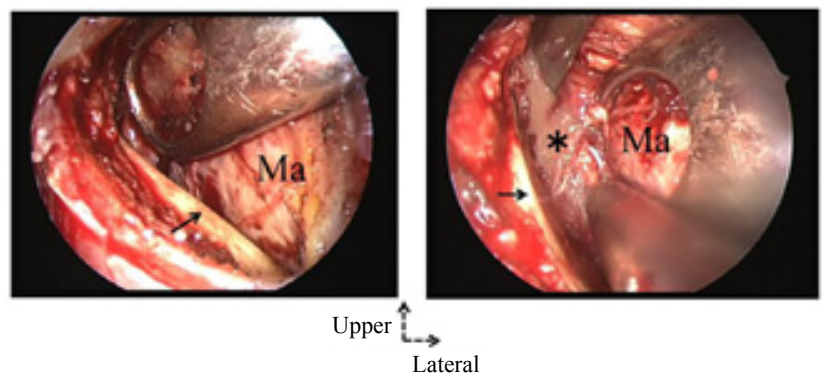

Figure 2: Endoscopic views of the intraoral approach. A) Dissection along the right mandibular ramus (arrow), reaching the lateral masticator space. B) After drainage of the abscess (star), located between the masseter (star) and periosteum of the mandibular ramus (arrow).

Ma: masticator muscle.

successfully performed. Second, quinsy tonsillectomy was performed, revealing an abscess cavity in the peritonsillar area.

The collected pus with swabs from the masticator space was immediately sent for bacteriological analysis, as previously described [7]. For aerobic bacteria, sheep blood, chocolate agar plates were incubated at $37^{\circ} \mathrm{C}$ in $5 \% \mathrm{CO}_{2}$ for 48 hours. For anaerobic bacteria, the specimen material was placed onto pre reduced vitamin K10 enriched brucella blood agar, and incubated in GasPac jars for 48 hours. Plates that showed growth were incubated for at least 7 days. Both of these aerobic and anaerobic cultures revealed growth of $S$. constellatus. Regarding antimicrobial susceptibility, the isolated S. constellatus show sensitive to penicillin, ampicillin, ceftriaxone, cefepime, meropenum, gatifloxacin, and linezolid according to the criteria of the Clinical and Laboratory Standards Institute [8].

The patient received intravenous injections of meropenem ( $3 \mathrm{~g} /$ day) for 16 days and clindamycin $(1.2 \mathrm{~g} /$ day) for 7 days, followed by sulbactam/ampicillin $(4.5 \mathrm{~g} /$ day) for 4 days because of the presumable drug eruption due to the meropenem. As shown in Table 1, cytopenia progressed postoperatively presumably because of infection and/ or adverse effects of the combined telaprevir therapy. Therefore, she also received a platelet transfusion of platelet concentrate (10 IU) on postoperative day 2 , and intravenous injections of granulocyte colonystimulating factor (G-CSF) $(75 \mu \mathrm{g})$ on postoperative days 6 and 7 .

A drainage tube inserted into the gingival mucosa was removed on postoperative day 4 , and the tracheal stoma was closed on postoperative day 10. She received rehabilitation for disturbance of the mouth opening, and gradually recovered after the operation. Enhanced CT taken11 days postoperatively revealed resolution of the abscess (data not shown), and the patient was discharged 23 days after admission.

\section{Discussion}

Deep head and neck infections show preferred pathways of spread, because they develop inside potential spaces formed and lined by layers of the deep neck fascia $[1,2]$. A peritonsillar abscess represents an infection between the tonsillar capsule and superior constrictor muscle [2]. Infections in this area usually spread outside, representing a parapharyngeal abscess. From the parapharyngeal space, infections have been reported to spread downward via the submandibular space (Figure 3). However, no previous reports, other than two cases we

\begin{tabular}{|l|c|c|c|c|c|c|}
\hline & Operative day & POD 2 & POD 5 & POD 7 & POD 9 & POD 13 \\
\hline WBC $(/ \mu \mathbf{l})$ & 3,300 & 1,900 & 1,400 & 3,200 & 8,400 & 5,900 \\
\hline Hb $(\mathbf{g} / \mathbf{d l})$ & 9.6 & 9.9 & 9.4 & 8.6 & 8.8 & 8.5 \\
\hline PLT $(/ \mu \mathbf{l})$ & 42,000 & 21,000 & 26,000 & 43,000 & 83,000 & 154,000 \\
\hline CRP $(\mathbf{m g} / \mathbf{d l})$ & 20 & 15.2 & - & 5.3 & 2.2 & 0.8 \\
\hline
\end{tabular}

POD, postoperative day; WBC, white blood cell count; Hb, hemoglobin;

PLT, platelet; CRP, C-reactive protein

Table 1: Temporal changes in blood tests.

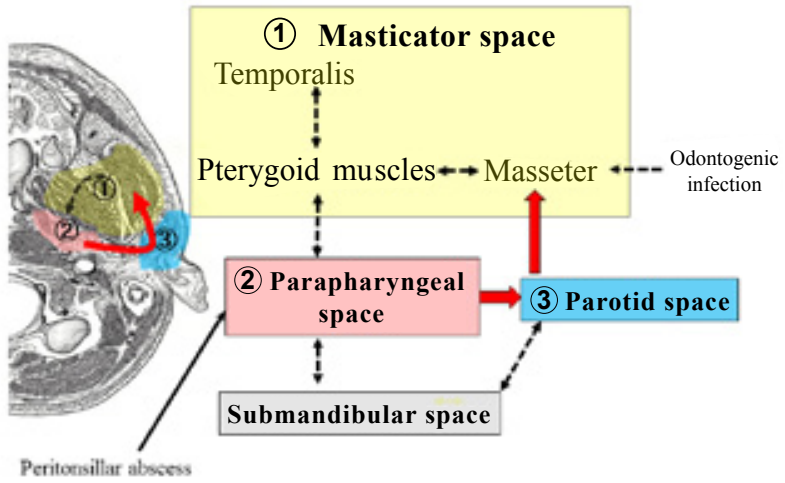

Figure 3: Anatomy of the masticator space and adjacent or communicating compartments, together with delineation of the associations of the masticator space with the surrounding head and neck spaces. Dotted lines show the preferred common pathways of intra- and extraspatial extensions of the infection. In terms of odontogenic infections, note that an abscess into the parapharyngeal space always spreads laterally through the masseter and medial pterygoid muscle. The unique extension in the current case from the parapharyngeal space to the masticator space isindicated by the red arrow. 
encountered [3], have been published describing this abscess extending into the masticator space.

The masticator space contains the masseter, pterygoid and temporalis muscles, and ramus and posterior part of the mandible [4]. Infections of this space are usually odontogenic, and these infections arising in the mandible spread first into the masseter and/or medial pterygoid muscles[4,5,9,10].The parapharyngeal space has been reported as a secondary site of spread for these infections[5].That is, infections from the pterygoid muscles sometimes spread medially to the parapharyngeal space (Figure 3). Therefore, all reports of odontogenic infections involving the parapharyngeal space have been reported to also be complicated with involvement of the medial pterygoid muscles $[4,5,9]$. Conversely, the present patienthad a peritonsillar abscess on the same side as the masticator abscess, and there were no CT findings of odontogenic infection intra operatively or involvement of the pterygoid muscles by infection. Another hypothetical route of extension to the masticator abscess from the parapharyngeal space would be anteriorly from the parotid space $[2,5,11]$. The present patient had involvement of infection into the parotid space; these findings were supported by the elevated blood amylase level. We can thus conclude that the current case, originating from the peritonsillar abscess, showed an unusual route of extension to the masticator space via the parotid space or along the surface of the mandibular ramus (Figure 3).

No background mechanisms have been confirmed to explain this peculiar route of extension, but the other two previous cases revealing similar extension from the parapharyngeal space to the masticator space had complications of systemic diseases [3].There were three underlying systemic conditions or diseases that might have been involved in the present patient. The first is the contribution of adverse effects of the antiviral HCV therapy, including blood cytopenia [12]. The HCV protease inhibitor telaprevir, administered in addition to peginterferon andribavirin, significantly increases the rate of sustained response in patients with genotype 1 chronic HCV infection [13,14]. The present symptoms started just 3 days after the $11^{\text {th }}$ round of the antiviral HCV therapy, coincidentally with the cytopenia. Whereas no previous reports have mentioned deep head and neck infections after combined telaprevir therapy, cytopenia might be attributed topathologies causing immune deficiency. Second, our patient had been prescribed prednisolone (20 $\mathrm{mg} /$ day) for 2 months because of the telaprevir-related dermatitis [15]. Patients affected by pathologies causing immune deficiency or who are taking immunosuppressive medications may display a unique clinical picture in comparison with patients without systemic disease [16,17]. These factors might have contributed to infectious aggravation. Third, our patient also suffered from fairly controlled diabetes mellitus (DM) attributed to the steroid therapy against telaprevir-related dermatitis. Systemic hyperglycemia results in derangement of the immune system including the neutrophil function, celluar immunity, and complement function $[1,16]$. Diabetes and advanced age have been reported as predisposing factors for more severe complications with spread of deep neck infection $[1,2,16]$. Whereas we have no confirmation regarding which of these three underlying factors mainly contributed to the unique extensions of the peritonsillar abscess in our patient, the combined anti-viral therapy contributed to the other two factors.

Surgical drainage remains the mainstay of treatment for deep neck abscess, especially for cases complicated by systemic disease $[1,16]$. In terms of incisional drainage for the masticator abscess, early reports described an external approach [10]. More recent descriptions have applied an intraoral approach, with dissection along the ramus [16]. The selection of these approaches has been the subject of much debate
[4,17]. Doxey et al. [4] advocated the former only for "simple" abscess limited to the masticator space. These authors applied an extra oral approach for more "complex" abscesses extending beyond the confines of the masticator space because of the difficulty in defining the true extent of infection. The usefulness of a modified Blair incision has been reported for accessing both the masticator and adjacent spaces [10], specifically for accessing the submandibular or lateral neck area. Conversely, endoscopy-assisted intraoral drainage has been discussed as useful, obviating the need for identifying facial nerve branches, avoiding unnecessary skin incision, and shortening anesthesia time [3]. As shown by the application of this approach for managing the infra temporal fossa [18], the endoscopic approach can overcome the difficulties in accessing narrow spaces such as the masticator space, allowing superior visualization with the ability to record operative findings.

Because the infection in the present patient did extend from the parapharyngeal space to the lateral masticator space along the mandible, and did not extend below the submandibular or lower cervical space, we selected the endoscopic-assisted intra-oral approach, followed by quinsy tonsillectomy. Successful drainage was achieved by combining the assessment of the original infectious region (parapharyngeal space) with the endpoint extension (lateral masticator space).

S. constellatus, isolated in the current case, is a member of the Streptococcus milleri group (SMG, also referred to as the "Streptococcus anginosus group") together with S.intermedius and S.anginosus. The SMG is found at various mucosal sites, including the oral cavity, genitourinary system, and gastrointestinal tract. However, this group of bacteria is also frequently encountered in invasive suppurative infections at various sites, including deep head and neck infections $[1,19,20]$. Furthermore, the authors also reported that SMG bacteria are the most frequent isolates of peritonsillar abscess [1,7]. The detailed pathogenesis of the SMG remains unclear, but infection of the mucus by normal flora is thought occur due to an imbalance between the organisms and the host immune defense in deep neck abscesses $[1,7,19]$. The presence of SMG bacteria would thus promote abscess formation, and consequently increase the need for surgical drainage [1].

Surgical drainage remains the mainstay of treatment for head and neck abscess, but administration of antimicrobials is also required [21]. Every patient who has a deep head and neck infection should be given initial empiric antibiotic therapy until culture and sensitivity results are available [22]. Length of antibiotic therapy for the peritonsillar and/or parapharyngeal abscess in older patients has been reported to range from 10 to 30 days, consisting of combined intravenous and oral routes [23]. First, we used meropenum in combination with clindamycin which would be highly effective against most anaerobes [22]. Drug resistance was not revealed to the isolated $S$. constellatus, consistent with the previous findings that few case reports have describes drug resistance to SMG $[1,24]$. Moreover, the patient suffered from drug eruption presumably because of meropenem, we thus changed the antibiotic therapy to sulbactam/ampicillin, and continued until the patient has been afebrile for 48 hours according to the previous review [22].

\section{References}

1. Hasegawa J, Hidaka H, Tateda M, Kudo T, Sagai S, et al. (2011) An analysis of clinical risk factors of deep neck infection. Auris Nasus Larynx 38: 101-107.

2. Maroldi R, Farina D, Ravanelli M, Lombardi D, Nicolai P (2012) Emergency imaging assessment of deep neck space infections. Semin Ultrasound CT MR 33: $432-442$.

3. Hidaka H, Ishida E, Suzuki T, Matsutani S, Tobayashi T (2013) Unusual 
Citation: Hidaka H, Suzuki T, Ishida E, RisakoKakuta, Yano H (2013) Unique Extension of Peritonsillar Abscess to the Masticator Space Encountered in the Early Phase of Antiviral Therapy for Hepatitis C. Clin Microbial 2: 117. doi:10.4172/2327-5073.1000117

parapharyngeal extension of peritonsillar abscess to the masticator space: successfullydrainaged by external and intraoral approaches. Ann OtolRhinolLaryngol, under revision.

4. Doxey GP, Harnsberger HR, Hardin CW, Davis RK (1985) The masticator space: the influence of CT scanning on therapy. Laryngoscope 95: 1444-1447.

5. Yonetsu K, Izumi M, Nakamura T (1998) Deep facial infections of odontogenic origin: CT assessment of pathways of space involvement. AJNR Am J Neuroradiol 19: 123-128.

6. Wang SM (2012) Peritonsillar abscess: cool "hot potato". Pediatr Neonatol 53 325-326.

7. Hidaka H, Kuriyama S, Yano H, Tsuji I, Kobayashi T (2011) Precipitating factors in the pathogenesis of peritonsillar abscess and bacteriological significance of the Streptococcus milleri group. Eur J Clin Microbiol Infect Dis 30: 527-532.

8. Pereira V, Lopes C, Castro A, Silva J, Gibbs P, et al. (2009) Characterization for enterotoxin production, virulence factors, and antibiotic susceptibility of Staphylococcus aureus isolates from various foods in Portugal. Food Microbiol 26: $278-282$.

9. Schuknecht B, Stergiou G, Graetz K (2008) Masticator space abscess derived from odontogenic infection: imaging manifestation and pathways of extension depicted by CT and MR in 30 patients. Eur Radiol 18: 1972-1979.

10. Newman MH Jr, Emley WE (1974) Chronic masticator space infection. Arch Otolaryngol 99: 128-131.

11. Al-Belasy FA (2005) Ultrasound-guided drainage of submasseteric space abscesses. J Oral Maxillofac Surg 63: 36-41.

12. Chopra A, Klein PL, Drinnan T, Lee SS (2013) How to optimize HCV therapy in genotype 1 patients: management of side-effects. Liver Int 33 Suppl 1: 30-34.

13. McHutchison JG, Everson GT, Gordon SC, Jacobson IM, Sulkowski M, et al. (2009) Telaprevir with peginterferon and ribavirin for chronic HCV genotype 1 infection. N Engl J Med 360: 1827-1838.
14. Zeuzem S, Andreone P, Pol S, Lawitz E, Diago M, et al. (2011) Telaprevir for retreatment of HCV infection. N Engl J Med 364: 2417-2428.

15. Roujeau JC, Mockenhaupt M, Tahan SR, Henshaw J, Martin EC, et al. (2013) Telaprevir-related dermatitis. JAMA Dermatol 149: 152-158.

16. Huang TT, Liu TC, Chen PR, Tseng FY, Yeh TH, et al. (2004) Deep neck infection: analysis of 185 cases. Head Neck 26: 854-860.

17. Akst LM, Albani BJ, Strome M (2005) Subacute infratemporal fossa cellulitis with subsequent abscess formation in an immunocompromised patient. Am J Otolaryngol 26: 35-38.

18. Prosser JD, Figueroa R, Carrau RI, Ong YK, Solares CA (2011) Quantitative analysis of endoscopic endonasal approaches to the infratemporal fossa Laryngoscope 121: 1601-1605.

19. Han JK, Kerschner JE (2001) Streptococcus milleri: an organism for head and neck infections and abscess. Arch Otolaryngol Head Neck Surg 127: 650-654.

20. Hirai T, Kimura S, Mori N (2005) Head and neck infections caused by Streptococcus milleri group: an analysis of 17 cases. Auris Nasus Larynx 32 $55-58$

21. Brook I (2009) Current management of upper respiratory tract and head and neck infections. Eur Arch Otorhinolaryngol 266: 315-323.

22. Vieira F, Allen SM, Stocks RM, Thompson JW (2008) Deep neck infection Otolaryngol Clin North Am 41: 459-483, vii.

23. Franzese CB, Isaacson JE (2003) Peritonsillar and parapharyngeal space abscess in the older adult. Am J Otolaryngol 24: 169-173.

24. Tracy M, Wanahita A, Shuhatovich Y, Goldsmith EA, Clarridge JE 3rd, et al. (2001) Antibiotic susceptibilities of genetically characterized Streptococcus milleri group strains. Antimicrob Agents Chemother 45: 1511-1514. 